\title{
A Management System for Improving Traffic Efficiency in Transportation Infrastructures
}

\author{
Y. Kritikou \\ G. Dimitrakopoulos \\ E. Dimitrellou \\ P. Demestichas \\ University of Piraeus \\ Department of Digital Systems 80, Karaoli Dimitriou str., 18534, Piraeus, GREECE
}

kritikou@unipi.gr

\begin{abstract}
The continuously increasing need for mobility has brought about not only significant facilities in several aspects of human initiative, but also growing traffic congestions, a phenomenon that leads at a short time level to unpleasant everyday situations, but in the long run also to the degradation of the level of quality of living in large cities. The management of traffic stands thus as a fundamental prerequisite for confronting those issues and enhancing transportation. This paper considers the concept of car pooling as a structured approach to this problem, by specifying, developing and validating a mobile-community-driven system for collaborative transportation, namely the "Transportation Management - Car Pooling System”. The system is capable of proposing optimal, reliable and secure community matches (taking into consideration personality features, talking interests, driving style, etc.), based on user profile and context information. The paper describes the Transportation Management - Car Pooling System, presenting its input parameters, decision making process and outcomes. Finally, indicative simulation results showcase its effectiveness.
\end{abstract}

\section{Keywords}

Car pooling, traffic management

\section{INTRODUCTION}

Current socio-economic circumstances in Europe impose an increased need for mobility. Most European cities are overcrowded with vehicles, facing a continuously growing volume of traffic. Traffic congestions, an everyday phenomenon, are basically caused by the large number of vehicles, moving or searching for a parking place [21]. In addition, they are often incurred by unpredicted accidents and emergencies, which are, at the same time, associated with serious injuries / fatalities.

These facts show that there are important inefficiencies related to transportation. Inefficiencies cause enormous losses of time (e.g., the period that drivers spend in traffic congestion or while they search for parking places), decreases in the level of safety for both, vehicles and pedestrians, high pollution, degradation of quality of life, and huge waste of non renewable fossil energy [9], [19], [21]. Those inefficiencies have brought up the necessity for developing systems for more efficient and safer mobility. In response to the above, transportation (also traffic) management has been lately attracting enormous research effort, being established as a key service that should be offered, in the area of transportation, by Information and Communication Technologies (ICT) [10], [13], [22].
A phenomenon that is quite aligned with the inefficiency of transportation is the continuously increasing level of utilization of vehicles (i.e. vehicles are used in more and more aspects of human initiative), linked to the minimization of vehicle passengers (i.e. vehicles carrying only the driver), due to the increase in vehicle ownerships [21]. Apparently, this causes a significant increase in traffic volumes. To address this drawback, only some independent solutions have been proposed in the United States, Germany, Greece and Great Britain [4], [5], [6], [7], [11], [15], [16], [18]. This paper builds on those proposals and presents an innovative, widely applicable approach that lies on the advances of telecommunications [21]. On one hand, internet community portals provide the means to bring people together sharing the same interests and needs in order to ease poor social fabric and remove interaction barriers. On the other hand, car pooling (sharing) services (most popular in the US [3], [5], [11]) are chosen as one of the solutions for improving transportation efficiency. As a definition, "car pooling is at least two people riding in a car. Each member would have made the trip independently if the carpool had not been there. Driver and passengers know before the trip that they will share the ride and at what time they will be leaving. Professional and/or commercial vehicles are excluded. Both the driver and the passenger(s) are considered as carpoolers”, as stated in [12][21]. With the vision to improve both, transportation and social fabric, the paper combines the concepts of communities, mobility and car pooling into one integrated, community driven, mobility solution. In particular, it presents an optimization method that leads to mobile-communitydriven, secure, collaborative transportation, which is capable of proposing optimal, as well as reliable and safe community matches (e.g., safe driving, talking interests, etc.) and routes, based on profile and context information in mobile situation contexts.

The contribution of this work is twofold:

First, it specifies and develops a mobile-community driven optimization strategy that proposes community matches and routes, based on user profile and context information and succeeds in reducing the overall traffic loads in transportation infrastructures, through exploiting advanced transportation management solutions.

Second, it delivers inter-disciplinary solutions that benefit from advanced ICT technologies and the sociology and psychology sciences, improving social fabric, since based on user profiles (drivers and passengers), people are connected, and communities are built out of these interactions. Through these communities people can share transportation and generally support each other.

In the light of the above, the structure of the paper is as follows: the next section presents the motivation for this work and 
describes in a high level fashion an optimization scheme that can efficiently manage transportation infrastructures through the concept of car pooling, namely the Transportation Management Car Pooling System (TM-CPS). Section 3 presents the TM-CPS in detail, whereas section 4 provides some indicative simulation results that showcase the system's efficiency. Finally, concluding remarks are drawn in section 5 .

\section{MOTIVATION, BUSINESS CASE AND HIGH-LEVEL DESCRIPTION}

\subsection{Motivation}

As previously mentioned, the necessity to eliminate the continuously growing volume of traffic is broadly accepted, since it involves versatile, everyday, unsatisfactory situations. A solution approach to this could be the concept of "car pooling", which suggests that the passenger and the driver may agree to share the same ride to a certain destination, based on an agreement upon specific criteria. The results of such an action are, on one hand to have reduced number of cars on the route, and on the other hand to significantly reduce the expenses for gas or parking (there is an agreement of sharing the car expenses between the passengers [1], [17], [20], [24]). In addition to the above, the overall energy consumed is significantly reduced, and therefore pollution and $\mathrm{CO} 2$ emissions become less. In this respect, car pooling contributes to the diminishment of atmospheric pollution, more than any state-imposed policy [1], [17], [20], [24]. Also, the participants may take turns through sharing their vehicles [5]. Last but not least, car pooling may also provide social connections in an increasingly disconnected society [25]. Aligned with the latter, online car pooling services offer new ways to make social connections through discussion sites and custom ride-sharing services.

An important measure that has been already applied in countries that car pooling is widely accepted (i.e. in twenty states of U.S.A.) is the designated car pool lanes on highways (usually called High-Occupancy Vehicle - HOV lanes), which make traveling faster [5], [19]. These lanes concern cars having more than 2 passengers. Researches have shown that such lanes operate in an encouragingly effective manner [5].

In the light of the above, car pooling seems an attractive concept that could contribute to a more intelligent management and improvement of transportation circumstances. In this respect, the next subsection indicates how a car pooling system could become of commercial use, while subsection 2.3 gives an overview of the TM-CPS which will be analyzed in the context of this paper.

\subsection{Business Case}

This section aims at exemplifying the role of car pooling and raising the issue of its application, to support transportation management in large cities that face congestion problems, through a system created for this purpose.

The business case assumes that a person wishing to reach a destination monitors a specific drivers' pool, seeking for a subset of drivers being directed towards the same place. The person in question (prospective passenger) interacts with all "candidate" drivers and examines whether an agreement can be established, regarding specific criteria that originate either in the passenger's preferences, or in the driver's intentions. Indicative parameters include on one hand the requested (by the passenger) and provided (by the driver) departure and destination point, as well as the itinerary's cost, and on the other hand, user profiles (age, gender, marital status, educational level and occupation, language, nationality, smoking habits, etc.). The driver-passenger pair selected is the one that results in the best matching of parameters.

A high-level scenario corresponding to the business case is shown in Figure 1.

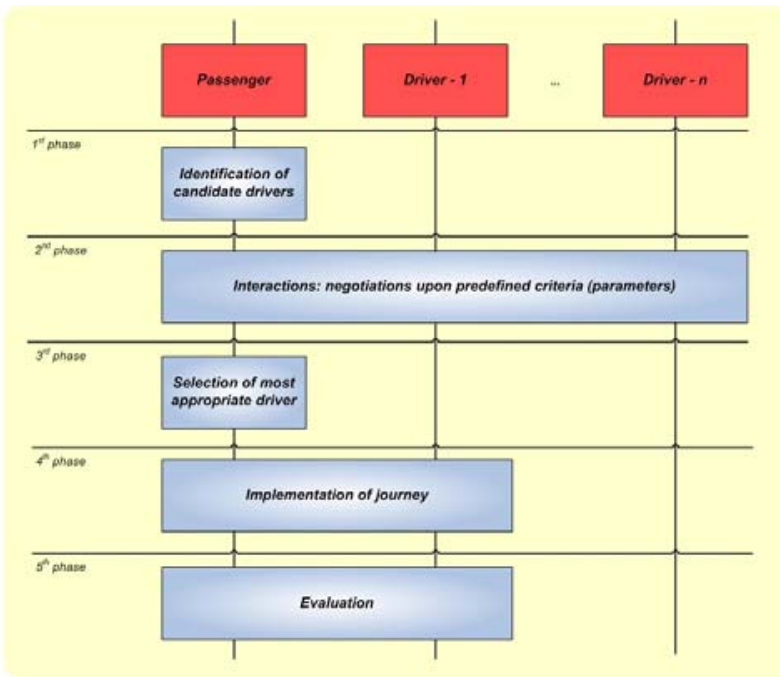

Figure 1: High-level car pooling scenario

The scenario can be either passenger-, or driver-initiated and evolves in five main phases. Examining the passenger-initiated scenario, in the first phase, the passenger seeks for drivers originating at a certain place and intending to reach a desired destination (the depiction of the driver-initiated scenario is omitted for brevity). In the second phase, the passenger interacts with the identified candidate drivers and negotiates upon a set of predefined criteria. In the third phase, the driver that satisfies most of the criteria is selected for the journey. The fourth phase refers to the implementation of the journey, while the final (fifth) phase, which takes place after the journey, consists in the evaluation provided by both parties of the journey, for future reference.

\subsection{High-Level TM-CPS Description}

The opportunity to share a vehicle, when having a similar itinerary and set of matching parameters, offered by the concept of car pooling, can be guaranteed through the existence of a system, accessible through the user's mobile phone or laptop/pc. In general, such a system has to take into consideration user's preferences and personal needs, as well as their changes as the time elapses, in order to adapt to them, taking in mind past preferences. Therefore, the system's two fundamental requirements are (i) personalization and (ii) adaptability, so as to effectively interact with the user [14].

Such a system is discussed herein, namely the TM-CPS. The TMCPS uses as input some user profile aspects, as well as some service aspects and aims at proposing optimal, reliable, secure and safe community matches and routes.

The TM-CPS is described in a high-level manner in Figure 2, while its main components are thoroughly analyzed in the next section. 


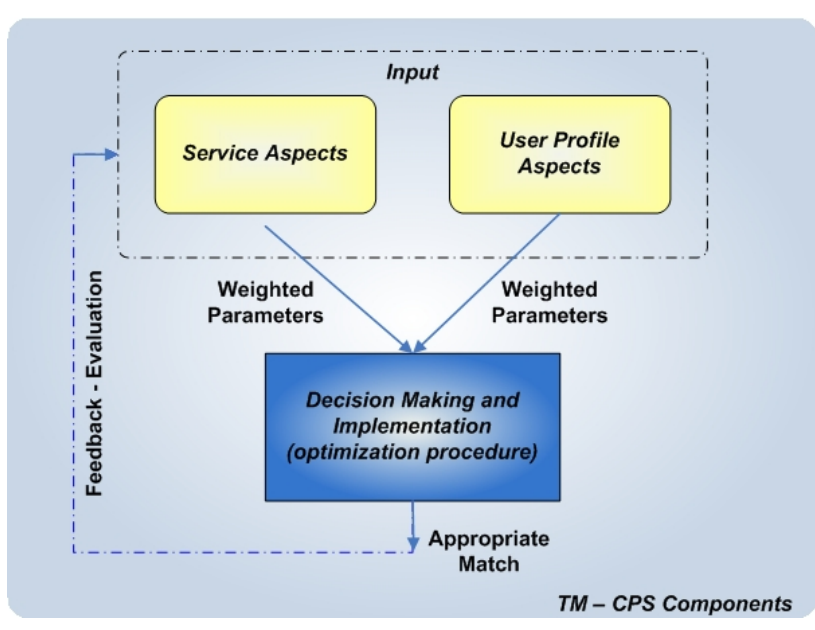

Figure 2: TM-CPS High-level description

\section{TM-CPS Detailed Analysis}

This section elaborates on the components of the TM-CPS presented in 2.3 and depicted on Figure 2, their operation and interactions [14], [26].

\subsection{User Profile Aspects}

This component stores all user-related data, namely personal information and preferences. It enables the system to know, immediately after the user logs on to the system, the identity of the user and the data the user has provided the system with. However, it should be noted that the user's preferences may change as the user makes use of the car pooling service. The component allows such amendments. The information handled through it is reflected on a list of parameters, discussed below.

User Profile Parameters. In general, the parameters that depict the data contained in the User Profile Aspects component include personal data and personal vehicle data. Personal vehicle data contain information on the vehicle that the user has and the characteristics that make it unique and identifiable by the passenger. These data are the number plate, the year the car was constructed, the year the user got his/ her driving license, the type and date of expiration of the car insurance and the brand of the car. Of course, personal vehicle data are optional, since a user of the system may not possess a car, yet can make use of the car pooling service, so they are just presented here for consistency and will remain unconsidered for the rest of the paper.

Personal data contain information regarding age, gender, marital status, educational level and occupation, language, nationality and whether the user is a smoker or not. More specifically, each user states his age, as well as the age category he wishes his copassenger to be in. Usually the gender of the user does not make any difference, yet sometimes the gender may help people in having common interests. Being married or single is of importance for users' matching. For instance, mothers may have many common interests regarding their children, than they would have while traveling with a student. A user's educational level is important, as it can create a more convenient atmosphere for cotravelers, as the chances of having something to discuss increase, and make the journey more pleasant. Occupation is another important parameter that affects the matching between users and creates higher possibility for it to succeed [5], [8]. The languages a user speaks may include him in a wider group of users and therefore have more chances of finding an appropriate match. Nationality is also a factor that influences the matching of users and their possibility to have a pleasant trip. Being a smoker or not may be very important for a user and affect at a great extend whether he will accept a matching or not. This is the reason for which in the beginning of the trip users establish some ground rules. In general, there has to be an agreement regarding smoking, music, food, drinks etc. Discussions are possible to irritate a user, as some may like quiet time in the morning, or a user may be sensitive to strong perfumes [7]. Rules are thus important to be set and agreed between users. In this respect, two additional parameters placed within this component (however not directly linked to user preferences) are (i) the itinerary's cost (to be discussed below) and (ii) the driving competence (deriving from others' evaluations, as will be shown in the sequel).

Parameters' Weight. Apart from the aforementioned parameters, a TM-CPS user needs to specify the importance he attributes to each of those parameters. This is achieved by valuing each of the parameters with a certain weight. Of course, it is possible that parameters could have the same weight for the user. For instance, a user may consider equally important that his co-passenger speaks English, as well as the fact that he is not a smoker. Practically, the user attributes each parameter with a value between 0 and 1 , with 0 implying that the parameter has a low importance for the user and 1 pointing at a high importance, respectively. In case a parameter has the same weight with another one, it is inferred that the user has equal interest in these parameter. Regarding the repetition of the match, the user does not attribute it with a certain weight, yet in the case that this user has traveled again with another user, the system can be informed whether the user is willing to share a ride again, based on the information extracted at the end of the itinerary.

As far as the cost of the itinerary is concerned, the user also specifies its weight. This means that the user is in position to state the level of expenses he can afford, or this is of no importance for him. The cost of the itinerary is calculated when a service request is made (this procedure is analyzed in 3.2). In order for the system to be aware of the gas expenses, in the beginning of the itinerary the driver sets the number of the kilometers, as well as whether the itinerary includes the tolls or not. Last but not least, the driver's driving competence and social behavior is evaluated by the passengers when the itinerary is completed. The value of these parameters is calculated as the mean of all passengers' evaluations. A summary of the parameters associated with the User Profile Aspects component and their notations, is provided in Table 1.

Table 1: User Profile Parameters

\begin{tabular}{|l|c|}
\hline \multicolumn{1}{|c|}{ Parameters } & Notation \\
\hline 1.Age & AG \\
\hline 2. Gender & GEN \\
\hline 3. Educational Level & EL \\
\hline 4. Family State & FS \\
\hline 5. Work & WOR \\
\hline 6. Smoking & SM \\
\hline 7. Language & LAN \\
\hline 8. Nationality & NT \\
\hline 9. Source & SR \\
\hline 10. Destination & DEST \\
\hline
\end{tabular}




\begin{tabular}{|l|c|}
\hline 11. Commuter Cost & CC \\
\hline 12. Evaluation & EST \\
\hline 12.1 Driving Skills & DS \\
\hline 12.2 Social Behavior & SB \\
\hline 12.3 Repeat Match & RM \\
\hline
\end{tabular}

\subsection{Service Aspects}

This component stores information about the service delivered to users. In particular, it is responsible for keeping records of the information regarding the service requests, the service requests that were satisfied and the users that were involved in the realization of the car pooling service. This means that when a user makes a car pooling service request, this request is received and decoded by the Service Aspects component, through identifying the critical information within the request (user id, departure point, destination point) and then passing this information to the User Profile Aspects component, in order to make the initial scanning of the user profiles and propose the potential matches.

The aforementioned critical information is reflected on a list of parameters, summarized in Table 2.

Table 2: Service Parameters

\begin{tabular}{|c|c|c|}
\hline $\begin{array}{c}\text { Driver } \\
\text { Parameters }\end{array}$ & $\begin{array}{c}\text { Matching } \\
\text { Parameters }\end{array}$ & $\begin{array}{c}\text { Passenger } \\
\text { Parameters }\end{array}$ \\
\hline \hline $\begin{array}{c}\text { Driver departure } \\
\text { point }\end{array}$ & Itinerary & $\begin{array}{c}\text { Passenger } \\
\text { departure point }\end{array}$ \\
\hline $\begin{array}{c}\text { Driver departure } \\
\text { time }\end{array}$ & Itinerary's cost & $\begin{array}{c}\text { Passenger } \\
\text { departure time }\end{array}$ \\
\hline $\begin{array}{c}\text { Driver } \\
\text { destination point }\end{array}$ & Pick-up point & $\begin{array}{c}\text { Passenger } \\
\text { destination point }\end{array}$ \\
\hline
\end{tabular}

A parameter that has to do with the itinerary is the passenger's starting point. The respective parameter for the driver is the driver's starting point, which is dependent on the itinerary he will follow to reach his destination. In other words, a matching takes place when passenger's departure and destination point are convenient for the driver's itinerary and departure and destination point. For instance, co-workers or people working in the same area of the city may agree on a car pooling service on a daily basis [8]. Moreover, itinerary's cost is agreed between users, so as they are both aware of the amount of money the passenger is going to compensate the driver. The cost is calculated taking into account the number of kilometers, as well as the fees spent on tolls, through a specific equation. The result of the equation represents the amount of money that the passenger will provide the driver and depends on the distance of the itinerary, and also the number and the cost of the tolls [17].

This equation can be defined as follows:

$$
\sum \text { cost }=\frac{(0.25 \square K m+2 \llbracket n)}{N}
$$

where $\mathrm{km}$ is the itinerary's distance (expressed in $\mathrm{km}$ ), $\quad N$ is the number of the passengers and $n$ expresses the times that tolls shall be paid throughout the itinerary. The cost of the tolls is set to $2 €$, yet it may change and the equation may formed accordingly.

Finally, it is important to be punctual and agree on how long the driver will wait for a passenger. The usual waiting time is 2 to 3 minutes [7].

\subsection{Optimization Method: Decision Making and Implementation}

This component receives the potential matches from the User Profile Aspects and the Service Aspects components. Its main responsibility is to make a decision amongst those matches, through an optimization process, which aims at maximizing the value of an objective function (OF) [23], whose variables are the profile and service parameters (see also Table 1, Table 2 and [2]). The OF value is calculated for every user based on the parameters and their respective weights. The driver for which the $\mathrm{OF}$ is maximized is the driver that best fits to the user requesting the service, and therefore the one that will be chosen among all candidates.

Let $x$ be the user, with $X$ denoting the set of users $(x \in X)$. Accordingly, let $d$ be the driver, where $D$ is the set of drivers ( $d \in D$ ). Each parameter (among the ones previously mentioned) is denoted as par, where $P A R$ is the set of parameters ( par $\in P A R$ ). In this respect, $W(x, p a r)$ is the weight for each parameter ( par ) for the user $x$ and $\operatorname{par}(d)$ is the value of the parameter that corresponds to the specific driver. The OF for the system is formed as followed:

$$
O F_{\text {System }}=\operatorname{Max}\left\{\sum_{d} K(x, d) \square \sum_{\text {par }} W(x, \operatorname{par}) \square \operatorname{par}(d)\right\}
$$

Where par $>0$ and $p a r=1, \ldots, N$. As can be observed, the $O F_{\text {System }}$ consists of the sum of the OFs of each driver $\left(O F_{d}\right)$ and the factor $K(x, d)$. The TM-CPS target is to identify the maximum amongst the OF values that correspond to each of the drivers. The $O F_{d}$ is given by the following equation:

$$
O F_{d}=\sum_{p a r} W(x, p a r) \square \operatorname{par}(d)
$$

Also, it holds that $K$ is a binary parameter which shows whether a driver is chosen by user or not. Specifically:

$$
K(x, d)=\left\{\begin{array}{l}
0, \text { if user } \mathrm{x} \text { does not choose driver } \mathrm{d} \\
1, \text { if user } \mathrm{x} \text { chooses driver } \mathrm{d}
\end{array}\right.
$$

This equation denotes that every user $x$ may select only one driver $d$ for each itinerary.

The former applies also to the reverse procedure, when a driver is seeking for a passenger, in order to use car pooling service. The scenarios that follow present this procedure and therefore the way OF works in order to find the best match for each user.

\subsection{Feedback - Evaluation}

As already mentioned, when registering on the platform, the user provides the system with their personal data and preferences. Yet, a change of user's preferences or personal data is possible, therefore the system will have to be able to update the according parameters and adapt to user's new preferences and needs. Of course, a user may update his profile by himself. On the other hand, changes in user's profile or preferences may be inferred through the evaluation procedure within the system. This procedure is done by the passengers concerning the drivers and vice versa, at the end of every ride. It provides the users (both the driver and the passenger) with the option to fill in a brief questionnaire, evaluating the driver/ passenger. The questions aim 
to extract the user's opinion on his co-passenger's social behavior, the driving competence and skills and whether he is willing to share the same ride with this driver or not.

Specifically, there are three categories of overall evaluation which a user can choose from: positive, neutral or negative. Also, the user may specify whether he is willing to share a ride again with the same user of the system. In this way the system may create, and each time update, a "+ " list for positively evaluated users and a "-“ list for negatively evaluated users. Driving style, driving competence and social behavior are some evaluation parameters which help the system to update the user profile, in order to create more successful matches in the future. In addition, each user can explain their evaluation through comments, giving e.g. details on the punctuality or reliability of the other member. Each user may have access to the evaluation user profile of a user, in which all received evaluations are collected. By viewing this profile, other users can view evaluations and profit from the experiences of other users [4]. It is important to make comments easily understandable and factual for other users, as other users may read evaluations. The purpose of the comments is to allow other members to hear of certain experiences and of course not to insult other users [4]. The update parameters are summarized in Table 3.

Table 3: System Update - Evaluation Parameters and their potential values

\begin{tabular}{|l|l|}
\hline \multicolumn{1}{|c|}{$\begin{array}{c}\text { System Update - } \\
\text { Evaluation Parameters }\end{array}$} & \multicolumn{1}{c|}{ Potential Values } \\
\hline \hline Overall evaluation on user & positive, neutral, negative \\
\hline $\begin{array}{l}\text { Willingness to share a ride } \\
\text { again }\end{array}$ & positive, neutral, negative \\
\hline Driving style & $\begin{array}{l}\text { calm, convulsive, environment } \\
\text { friendly and gas saving }\end{array}$ \\
\hline Driving competence & good, efficient, dangerous \\
\hline Social behavior & $\begin{array}{l}\text { pleasant, friendly, annoying, } \\
\text { rude }\end{array}$ \\
\hline
\end{tabular}

\section{Indicative Simulation Results}

This section contains indicative results that derive from the utilization of the TM-CPS to a simulated transportation infrastructure environment, so as to showcase its efficiency.

Two scenarios will be presented. The scenarios are user-driven, in that they are differentiated based on user preferences. The first one presents the typical paradigm of a car pooling service request, while the second one serves as an example for the case when a user values extremely high a certain parameter (the cost, in our case).

\subsection{Scenario 1: Regular service request}

We consider user Mary, who has already registered on the TMCPS and therefore disposes a unique identity in the system. Her starting point is SP-A and her destination point is DP-A, an itinerary of 18 kilometers $(\mathrm{km})$. At the same time, three (3) drivers make a car pooling system request, making the system aware that they are going to follow the itinerary SP-A to DP-A.

Mary is supposed to have already filled her personal profile, stating the weights of parameters and also depicting her personal preferences on the driver or passenger (in case she is the driver). Table 4 presents the parameters and their respective weights (viewed from both), the passenger and the driver side. In the sequel, following the procedure discussed, the TM-CPS is responsible for deciding upon the best possible match.

Table 4: Scenario 1 - Parameters and their respective weights

\begin{tabular}{|c|c|}
\hline Parameter & Weight \\
\hline Age & 0,09 \\
\hline $13-17$ & 0,1 \\
\hline $18-24$ & 0,25 \\
\hline $25-34$ & 0,4 \\
\hline $35-44$ & 0,16 \\
\hline $45-54$ & 0,05 \\
\hline $55-64$ & 0,02 \\
\hline $65-70$ & 0,02 \\
\hline Gender & 0,02 \\
\hline Male & 0,5 \\
\hline Female & 0,5 \\
\hline Educational Level & 0,07 \\
\hline Higher & 0,6 \\
\hline Medium & 0,2 \\
\hline Low & 0,2 \\
\hline Marital Status & 0,05 \\
\hline Married & 0,3 \\
\hline Single & 0,7 \\
\hline Occupation & 0,07 \\
\hline Employed & 0,4 \\
\hline Unemployed & 0,05 \\
\hline Housewife & 0,05 \\
\hline Student/ Pupil & 0,5 \\
\hline Smoking & 0,25 \\
\hline Yes & 0,1 \\
\hline No & 0,9 \\
\hline Language & 0,02 \\
\hline English & 0,3 \\
\hline French & 0,3 \\
\hline Greek & 0,3 \\
\hline Other & 0,1 \\
\hline Nationality & 0,05 \\
\hline English & 0,8 \\
\hline Other & 0,2 \\
\hline \multicolumn{2}{|l|}{ Evaluation } \\
\hline Driving Competence & 0,25 \\
\hline Good & 0,5 \\
\hline Medium & 0,35 \\
\hline Low & 0,15 \\
\hline Social Behavior & 0,08 \\
\hline Good & 0,8 \\
\hline Medium & 0,1 \\
\hline Low & 0,1 \\
\hline Itinerary Cost & 0,05 \\
\hline Economic & 0.6 \\
\hline Non-economic & 0.4 \\
\hline \multicolumn{2}{|l|}{ Repeat Match } \\
\hline (NMB) No Match Before & \\
\hline Yes & \\
\hline No & \\
\hline
\end{tabular}

Specifically, taking into consideration the data provided by Table 4, Error! Not a valid bookmark self-reference. is formed in order to provide reference to the parameters and their weights for 
each of the three suggested candidate drivers, in order to showcase their differences.

Table 5: Scenario 1 - Candidate Drivers’ Parameters and their Values

\begin{tabular}{|c|c|c|c|}
\hline Parameters & George & Kate & Nicolas \\
\hline Age & 29 yrs $(0,4)$ & 26 yrs $(0,4)$ & 34 yrs $(0,4)$ \\
\hline Gender & Male $(0,5)$ & Female $(0,5)$ & Male $(0,5)$ \\
\hline $\begin{array}{l}\text { Educational } \\
\text { Level }\end{array}$ & Medium $(0,2)$ & Higher $(0,6)$ & Higher $(0,6)$ \\
\hline $\begin{array}{l}\text { Marital } \\
\text { Status }\end{array}$ & Married $(0,3)$ & Single $(0,7)$ & Single $(0,7)$ \\
\hline Occupation & $\begin{array}{l}\text { Employed } \\
(0,4)\end{array}$ & Student $(0,5)$ & $\begin{array}{l}\text { Employed } \\
(0,5)\end{array}$ \\
\hline Smoker & No $(0,9)$ & Yes $(0,1)$ & Yes $(0,1)$ \\
\hline Language & English $(0,3)$ & English $(0,3)$ & English $(0,3)$ \\
\hline Nationality & English $(0,8)$ & English $(0,8)$ & English $(0,8)$ \\
\hline Departure & SP-A & SP-A & SP-A \\
\hline Destination & DP-A & DP-A & DP-A \\
\hline $\begin{array}{l}\text { Itinerary } \\
\text { Cost }\end{array}$ & $\begin{array}{l}\text { Non - } \\
\text { Economic } \\
(0,4)\end{array}$ & $\begin{array}{l}\text { Economic } \\
(0,6)\end{array}$ & $\begin{array}{l}\text { Non- } \\
\text { Economic } \\
(0,4)\end{array}$ \\
\hline \multicolumn{4}{|l|}{ Evaluation } \\
\hline $\begin{array}{l}\text { 1. Driving } \\
\text { Competence }\end{array}$ & Good $(0,5)$ & Good $(0,5)$ & $\begin{array}{l}\text { Medium } \\
(0,35)\end{array}$ \\
\hline $\begin{array}{l}\text { 2. Social } \\
\text { Behavior }\end{array}$ & Good $(0,8)$ & Medium $(0,1)$ & Good $(0,8)$ \\
\hline $\begin{array}{l}\text { 3. Repeat } \\
\text { Match }\end{array}$ & NMB & NMB & Yes \\
\hline
\end{tabular}

In addition, Figure 3 depicts the weights of the parameters with regards to Mary's preferences.

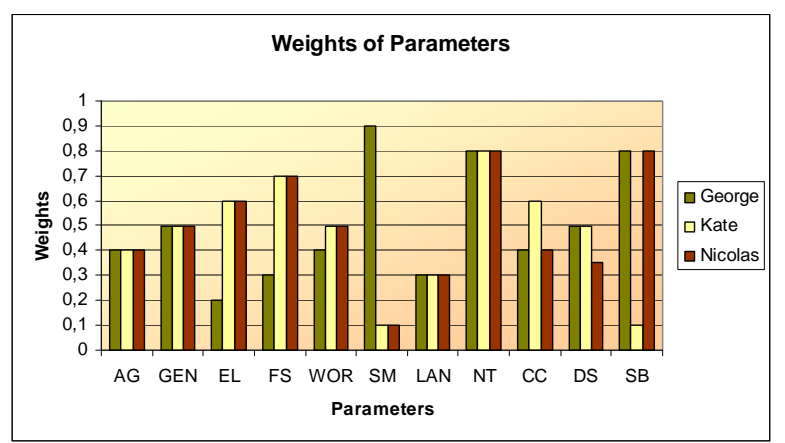

Figure 3: Scenario 1 - Parameters weights in combination with passenger's preferences

For every candidate driver, there is a respective OF value, based on the parameters and their weights, as presented in 3.3 and equation(3)

More specifically,

$$
\begin{aligned}
\mathrm{OF}_{\text {George }} & =\sum_{\text {par }} W(x, \operatorname{par}) \square \operatorname{par}(d)= \\
& 0,09 \square 0,5+0,02 \square 0,5+0,07 \unrhd 0,2+0,05 \square 0,3+0,07 \unrhd 0,4+ \\
& 0,25 \square 0,9+0,02 \square 0,3+0,05 \square 0,8+0,05 \square 0,4+0,25 \square 0,5+0,08 \square 0,8 \\
& =0,583
\end{aligned}
$$

Similarly,

$$
\begin{aligned}
\mathrm{OF}_{\text {Kate }}= & \sum_{p a r} W(x, \operatorname{par}) \square \operatorname{par}(d)= \\
& 0,09 \square 0,4+0,02 \square 0,5+0,07 \square 0,6+0,05 \square 0,7+0,07 \square 0,5+ \\
& 0,25 \square 0,1+0,02 \square 0,3+0,05 \square 0,8+0,05 \square 0,6+0,25 \square 0,5+0,08 \square 0,1 \\
& =0,392
\end{aligned}
$$

and

$$
\begin{aligned}
\mathrm{OF}_{\text {Nicolas }} & =\sum_{\text {par }} W(x, \operatorname{par}) \square \operatorname{par}(d)= \\
& 0,09 \square 0,4+0,02 \square 0,5+0,07 \unrhd 0,6+0,05 \square 0,7+0,07 \square 0,5+ \\
& 0,25 \square 0,1+0,02 \square 0,3+0,05 \square 0,8+0,05 \square 0,4+0,35 \square 0,5+0,08 \square 0,8 \\
& =0,4005
\end{aligned}
$$

Replacing the respective factors to equation(3) and taking into account equation(4), we construct the following equation:

$$
\begin{aligned}
\text { OF } & =\operatorname{Max}\left\{\sum_{d} K(x, d) \square \sum_{\text {par }} W(x, \text { par }) \square \operatorname{par}(d)\right\} \\
& =\operatorname{Max}\{1 \square 0,583+0 \sqsubset 0,392+0 \square 0,4005\} \\
& =0,583
\end{aligned}
$$

Based on the results, what can be concluded is that the TM-CPS will decide in favor of Mary and George, as George's OF value is the highest, among the three candidate drivers. It may also be observed that despite the fact that Kate and Nicolas comply with Mary's preferences as far as age, educational level and marital status are concerned, driver George is selected as more appropriate, as Mary thinks of driving competence and smoking as more important factors to make the match.

Figure 4 graphically presents the OF values of the 3 candidate drivers.

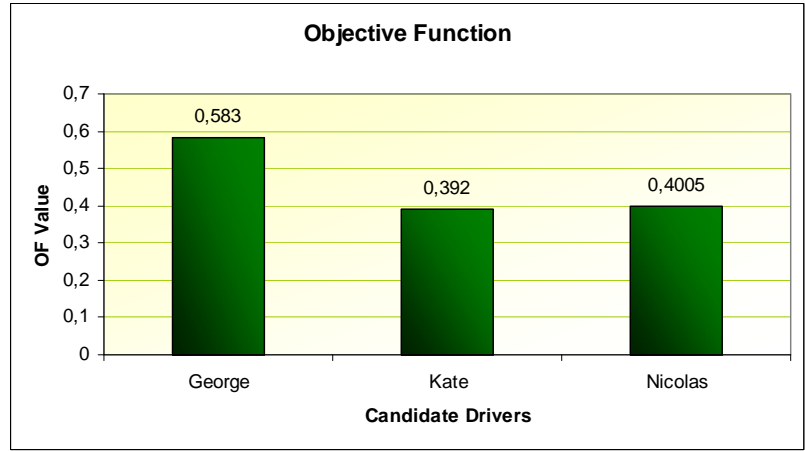

Figure 4: Scenario 1 - Objective function value for each candidate user

Furthermore, let it be noted that using equation (1), we can calculate the cost of the itinerary, which in this case is 2.25€.

When the selection of the users that will share the ride has been finalized, they contact each other by telephone, e-mail or SMS in order to set the details of the itinerary, namely the time of the pick-up, the pick-up point, the destination point, the maximum time of waiting at the pick-up point and the cost of the itinerary.

Of course, candidate drivers and passengers have the right to reject a potential match, as they will be able to view their 
candidate's match profile. In case one the users rejects a match, then the next choice is offered as a solution.

\subsection{Scenario 2: Cost - driven scenario}

In this case, user Thomas (a driver now) wishes to drive an itinerary of $463 \mathrm{~km}$, having as starting point the SP-K and destination point the DP-L. This means that the itinerary is an interurban itinerary and it definitely involves tolls. Driver Thomas specifies the day and time of departure, the possible stops he is going to make during the itinerary and the number of tolls he is going to pay:

$>$ Starting Point: SP-K

$>$ Destination Point: DP-L

Kilometers: $463 \mathrm{Km}$

Number of tolls: 5

In such a long itinerary it seems rational that, more than any case, the cost is important to the users, as it is higher than any other itinerary. The weights of parameters for Thomas are presented in Table 6. As it may be observed, the driver thinks of the cost as a very important factor of the itinerary. Another point to underline is that in the evaluation part, the sub-parameter "Driving Competence" is not taken into consideration (it is equal to zero). This is because in this case the driver is the one that looks for a co-passenger and not vice versa. Therefore, it is of no importance whether the passenger is a competent driver or a driver at all, since he is not going to use such skills. There are three users of the system that wish to make the same itinerary as Thomas and seem to be close to the characteristics that the driver has applied for.

Table 6: Scenario 2 - Parameters and their respective weights

\begin{tabular}{|c|c|}
\hline \multicolumn{1}{|c|}{ Parameter } & Weight \\
\hline Age & 0,1 \\
\hline $13-17$ & 0,15 \\
\hline $18-24$ & 0,3 \\
\hline $25-34$ & 0,3 \\
\hline $35-44$ & 0,1 \\
\hline $45-54$ & 0,05 \\
\hline $55-64$ & 0,05 \\
\hline $65-70$ & 0,05 \\
\hline Gender & 0,02 \\
\hline Male & 0,5 \\
\hline Female & 0,5 \\
\hline Educational Level & 0,09 \\
\hline Higher & 0,5 \\
\hline Medium & 0,35 \\
\hline Low & 0,15 \\
\hline Marital Status & 0,04 \\
\hline Married & 0,45 \\
\hline Single & 0,55 \\
\hline Occupation & 0,08 \\
\hline Employed & 0,2 \\
\hline Unemployed & 0,25 \\
\hline Housewife & 0,05 \\
\hline Student/ Pupil & 0,5 \\
\hline Smoking & 0,15 \\
\hline Yes & 0,6 \\
\hline No & 0,4 \\
\hline
\end{tabular}

\begin{tabular}{|c|c|}
\hline Language & \multicolumn{1}{l|}{0,02} \\
\hline English & 0,3 \\
\hline French & 0,3 \\
\hline Greek & 0,3 \\
\hline Other & 0,1 \\
\hline Nationality & 0,05 \\
\hline English & 0,65 \\
\hline Other & 0,35 \\
\hline Evaluation & \\
\hline Driving Competence & 0,0 \\
\hline Good & 0,0 \\
\hline Medium & 0,0 \\
\hline Low & 0,0 \\
\hline Social Behavior & 0,1 \\
\hline Good & 0,8 \\
\hline Medium & 0,1 \\
\hline Low & 0,1 \\
\hline Itinerary Cost & 0,35 \\
\hline Economic & 0,1 \\
\hline Non-economic & 0,9 \\
\hline Repeat Match & \\
\hline (NMB) No Match Before & \\
\hline Yes & \\
\hline No & \\
\hline present & \\
\hline
\end{tabular}

Table 7 presents the weights that correspond to the values of parameters for each candidate user, as formed according to Thomas' preferences and priorities, expressed in Table 6.

Table 7: Scenario 2 - The weights of the parameters for the candidate passengers

\begin{tabular}{|c|c|c|c|}
\hline Parameters & Margaret & Amy & Jim \\
\hline Age & 23 yrs $(0,3)$ & 53 yrs $(0,05)$ & 31 yrs $(0,3)$ \\
\hline Gender & Female $(0,5)$ & Female $(0,5)$ & Male $(0,5)$ \\
\hline $\begin{array}{l}\text { Educational } \\
\text { Level }\end{array}$ & Higher (0.5) & $\begin{array}{l}\text { Medium } \\
(0,35)\end{array}$ & Higher $(0,5)$ \\
\hline $\begin{array}{l}\text { Marital } \\
\text { Status }\end{array}$ & Single $(0,55)$ & $\begin{array}{l}\text { Married } \\
(0,45)\end{array}$ & $\begin{array}{l}\text { Single } \\
(0,55)\end{array}$ \\
\hline Occupation & Student $(0,5)$ & $\begin{array}{l}\text { Housewife } \\
(0,05)\end{array}$ & $\begin{array}{l}\text { Employed } \\
(0,2)\end{array}$ \\
\hline Smoker & Yes $(0,6)$ & No $(0,4)$ & Yes $(0,6)$ \\
\hline Language & English $(0,3)$ & English $(0,3)$ & English (0.3) \\
\hline Nationality & English $(0,65)$ & English $(0,65)$ & $\begin{array}{l}\text { English } \\
(0,65)\end{array}$ \\
\hline Departure & SP-K & SP-K & SP-K \\
\hline Destination & DP-L & DP-L & DP-L \\
\hline $\begin{array}{l}\text { Itinerary } \\
\text { Cost }\end{array}$ & $\begin{array}{l}\text { Economic } \\
(0,1)\end{array}$ & $\begin{array}{l}\text { Non - } \\
\text { Economic } \\
(0,9)\end{array}$ & $\begin{array}{l}\text { Economic } \\
(0,1)\end{array}$ \\
\hline \multicolumn{4}{|l|}{ Evaluation } \\
\hline $\begin{array}{l}\text { 1. Driving } \\
\text { Competence }\end{array}$ & _ & - & - \\
\hline $\begin{array}{l}\text { 2. Social } \\
\text { Behavior }\end{array}$ & Good $(0,8)$ & Good $(0,8)$ & Good $(0,8)$ \\
\hline $\begin{array}{l}\text { 3. Repeat } \\
\text { Match }\end{array}$ & NMB & NMB & Yes \\
\hline
\end{tabular}

Then, based on the parameters and their weights, we can calculate the OF's value for the three candidate passengers, using the equation(2), and following the example of the first scenario. 
Figure 5 presents the values of the parameters for every candidate passenger, while Figure 6 presents the OF values for all three candidate passengers.

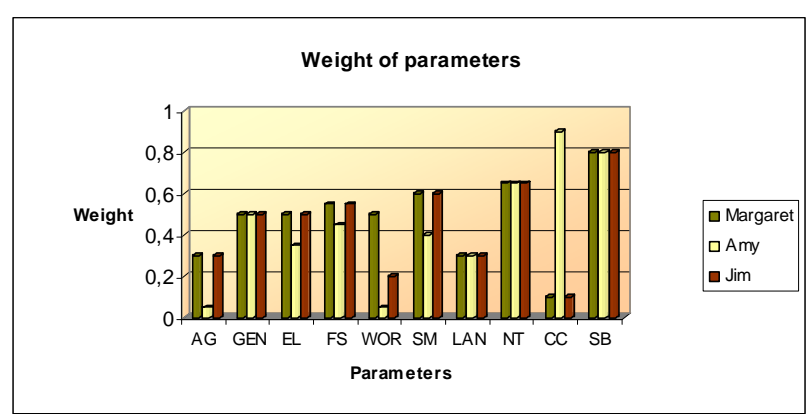

Figure 5: Scenario 2 - Parameters weights in combination with driver's preferences

Out of the three candidates, Margaret and Jim have almost all parameters' values similar and very high. This means that they have high chances to match with Thomas. Thus, it would be sensible that the system would have to choose between one of the two passengers (Margaret or Jim) to travel with. Yet, since Thomas thinks that the cost is the most important factor, Amy will be chosen, as she is the user that has the same opinion with Thomas, that is a non-economic itinerary, and Thomas attributes a high importance to this parameter (providing a high value). This means that Thomas would like to travel through the most expensive and yet safer manner, which is through the National Highways.

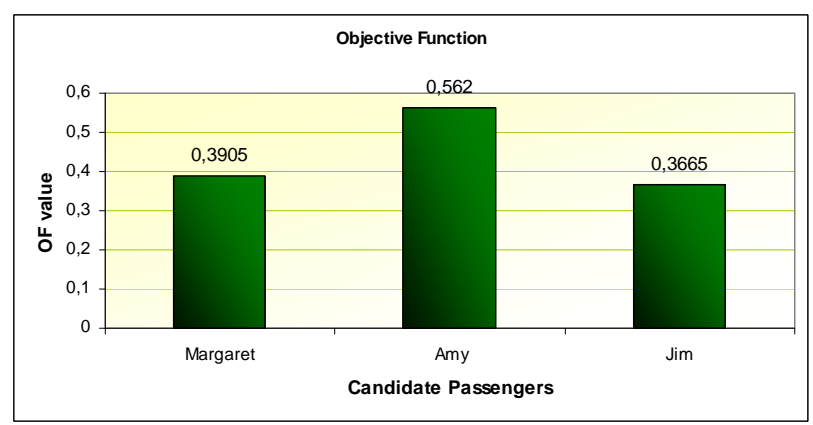

Figure 6: Scenario 2: Objective function value for each candidate user

Figure 6 shows in a graphical way the OF values of the 3 candidate drivers. Moreover, using equation (1), we can calculate the cost of the itinerary, which in this case is $\mathbf{6 2 , 8 7} €$.

This is a typical example showing that the weights can catalytically affect the results of the $\mathrm{OF}$ value calculation. However, the driver is in position to reject the candidate passenger with the highest $\mathrm{OF}$ value and choose the passenger that has resulted to the second highest OF value, as also previously mentioned.

\section{Conclusions and Future Work}

The continuously increasing need for mobility has brought about significant changes in transportation infrastructures, usually associated with unpleasant traffic congestions. Those unpleasant phenomena raise the necessity for more efficient and safer mobility. A valid option is to efficiently manage traffic and lies in the concept of car pooling, which envisages that drivers and passengers can share an itinerary, increasing the number of passengers per vehicle, while decreasing the number of vehicles in the route. In this respect, the paper has proposed a system, namely the "Transportation Management - Car Pooling System" (TM-CPS), which is capable of proposing optimal, reliable and secure community matches, based on profile and context information. Apart from the detailed description of the TM-CPS components, the paper has also gone through extensive simulations, the results of which are more than encouraging in proving the TM-CPS's capability to be applied in real time transportation infrastructures.

In general, the TM-CPS represents a simple, while absolutely effective means to eliminate traffic congestions, contributing at the same time to the reduction of environmental pollution, as well as to the enhancement of social relations amongst strangers. As the concept of car pooling is envisaged to attract significant attention in the near future, more and more organizations will target the utilization of management systems, such as the TMCPS.

A potential improvement to the system could consist in a further analysis of cost factors that should be taken into account, with this denoting the design and development of advanced, composite objective functions that should be optimized when proposing matches. Furthermore, another aspect to be analyzed is the creation of user groups, by taking into account user history preferences (the parameter used in this system "Repeat Match", as well as personal characteristics of the user (area of residence, area of work, etc). In this way, the system will search on a first level within the group and if not finding a match, then it will proceed on the broader level of the system as a whole. Additionally, as a future work, the TM-CPS shall be incorporated in larger transportation management systems. This implies the consideration of work on distributed, cognitive functionality for the management of transportation, in terms of combining the concept of car pooling with other legacy and novel methods, such as vehicles redirections or traffic lights amendment, according to the management system's commands.

\section{Acknowledgment}

This work was performed in project E3 which has received research funding from the Community's Seventh Framework programme. This paper reflects only the authors' views and the Community is not liable for any use that may be made of the information contained therein. The contributions of colleagues from E3 consortium are hereby acknowledged

\section{References}

[1] American Automobile Association Website. http://www.aaanewsroom.net/main/Default.asp?CategoryID $=5$ (Accessed March 2008).

[2] Black P. Algorithms and Theory of Computation Handbook, "objective function", in Dictionary of Algorithms and Data Structures [online], U.S. National Institute of Standards and Technology. CRC Press LLC. December 2004. 
[3] Burris M W and Winn J R. Slugging in Houston: Casual Carpool Passenger Characteristics. Transportation Research Board 85th Annual Meeting, 2006.

[4] Car-Pool UK Website. http://www.carpool.co.uk/hilfe.php?landnr=GB\&partner=\&abfrg=2\&thema $=8 \&$ (Accessed March 2008).

[5] Carpoolconnect Website. http://carpoolconnect.com/carpool (Accessed March 2008).

[6] Carpooling Website. http://www.carpooling.gr/ (Accessed March 2008).

[7] Carpooltool Website. http://www.carpooltool.com/en/my/tips.php (Accessed March 2008).

[8] Cross B. Employers Go For Car Pooling. ITS International Journal; Vol. 11 No. 3, 2005.

[9] Division of Resources Management (DARM). Carpooling and You. Public domain document, Florida, U.S., January 2005.

[10] Dey A and Abowd G. Towards a Better Understanding of Context and Context-Awareness. Technical Report GITGVU-99-22, Georgia Institute of Technology, College of Computing, 3, 5, 1999.

[11] Erideshare Website. http://www.erideshare.com/ (Accessed March 2008).

[12] Icaro, 1999. Universitaet fuer Bodenkultur - Institute for Transport Studies, ICARO Increase Of CAR Occupancy through innovative measures and technical instruments, Final Report, Availability P, Vienna, Project Funded by the European Commission under the Transport RTD Programme of the 4th Framework Programme, November 1999.

[13] Kephart J and Chess D. The vision of autonomic computing. IEEE Computer, Vol. 36, No.1, pp. 41-50, January 2003.

[14] Kritikou Y, Demestichas P, Adamopoulou E, Demestichas K, Paradia M. User Profile Modeling in the Context of Web - Based Learning Management Systems. Journal of Network and Computer Applications, Elsevier, 2007.

[15] Liftpool DE Website. http://www.liftpool.de (Accessed March 2008).

[16] Liftpool UK Website. http://www.liftpool.co.uk/ (Accessed March 2008)
[17] Municipality of Anchorage, Alaska Website. http://www.muni.org/transit1/tips.cfm (Accessed March 2008).

[18] Nationalcarshare Website. http://www.nationalcarshare.co.uk/ (Accessed March 2008).

[19] Poole R W Jr and Balaker T. Virtual Exclusive Busways: Improving Urban Transit While Relieving Congestion. Policy Study 337, Reason Foundation, September 2005.

[20] Queensland Government Website. http://www.transport.qld.gov.au/Home/General_information/ Travelsmart/How_to_travelsmart/Car_pooling/ (Accessed March 2008).

[21] Steger-Vonmetz DI C. Improving modal choice and transport efficiency with the virtual ridesharing agency. Proceedings of the 8th International, IEEE Conference on Intelligent Transportation Systems, Vienna, Austria, September 13-16, 2005.

[22] Thomas R, Friend D, DaSilva L and McKenzie A. Cognitive networks: adaptation and learning to achieve end-to-end performance objectives. IEEE Commun. Mag., Vol. 44, No. 12, December 2006.

[23] Tsagkaris K, Dimitrakopoulos D, Saatsakis A, Demestichas P. Distributed Radio Access Technology Selection for Adaptive Networks in High-Speed, B3G Infrastructures. International Journal of Communication Systems, October 2007.http://egnatia.ee.auth.gr/ imatz/ann_chapter1.html

[24] University of Maine Website. http://www.umaine.edu/parking/carpooling\%20R\&R.html (Accessed March 2008).

[25] Watch HA EU. Internet - based Car Pooling in Greece. Source: CENTRICO, 27/7/2006 http://www.haeuwatchits.info/press/press_detail.asp?pid=13 5\&aid=345 (Accessed March 2008).

[26] Zakaria MR, Moore A, Stewart CD and Brailsford TJ. 'Pluggable' user models for adaptive hypermedia in education. Proceedings of the Fourteenth ACM Conference on Hypertext and Hypermedia 2003; Nottingham, UK, pp. 170 - 171, 2003. 